\title{
Introduction to the Guideline, and General Principles of Acute Migraine Management
}

\author{
Irene Worthington ${ }^{1}$, Tamara Pringsheim ${ }^{3}$, Marek J. Gawel ${ }^{1,8,9}$, \\ Jonathan Gladstone ${ }^{1,2}$, Paul Cooper ${ }^{4}$,Esma Dilli ${ }^{5}$, Michel Aube ${ }^{6}$, \\ Elizabeth Leroux ${ }^{7}$, Werner J. Becker ${ }^{3}$ on behalf of the Canadian Headache \\ Society Acute Migraine Treatment Guideline Development Group
}

\begin{abstract}
Objectives: To provide an overview of the objectives and target population of the guideline, and to review the general principles of acute pharmacological migraine therapy. Methods: A general literature review and several consensus groups were used to formulate an expert consensus for the general use of acute migraine medications. Results: The objective of the guideline is to assist the physician in choosing an appropriate acute migraine medication for an individual with migraine, and thereby to reduce migraine-related disability. The target population includes adults with episodic migraine (patients with migraine headache $<15$ days/month). This guideline is intended primarily for physicians who treat patients with migraine. Other health professionals may also find this guideline helpful. Acute migraine therapy should be considered for the great majority of patients with migraine. A specific acute medication is chosen based on evidence for efficacy, tolerability, migraine attack severity, patient preference, and on the presence of co-existing disorders. General principles of acute migraine therapy include that the response of a patient to any given medication cannot be predicted with certainty, and that treatment early in the attack is generally more effective than treatment later once the migraine attack is fully developed. A suitable treatment approach (stratified or stepped approaches) and drug formulation (injection, tablet, wafer, powdered formulation, or nasal spray) should be chosen based on patient clinical features. Excessively frequent use of acute medications (medication overuse) should be avoided. Two or more acute medications can be combined if necessary. Conclusions: This guideline provides evidence-based advice on the use of acute medications for migraine, and should provide useful guidance for acute migraine therapy to both health professionals and patients.
\end{abstract}

RÉSUMÉ: Introduction aux lignes directrices et aux principes généraux du traitement de la crise aiguë de migraine. Objectifs : Le but de cet article est de fournir un aperçu des objectifs et de la population ciblée par les lignes directrices et de revoir les principes généraux du traitement pharmacologique de la crise aiguë de migraine. Méthode : Nous avons effectué une revue de littérature et utilisé plusieurs groupes de consensus pour formuler un consensus expert concernant l'utilisation générale des médicaments pour traiter la crise aiguë de migraine. Résultats : L'objectif des lignes directrices est d'aider le médecin à choisir un médicament approprié pour traiter la crise aiguë de migraine chez un individu présentant de la migraine et ainsi diminuer l'invalidité due à la migraine. La population cible est constituée d'adultes présentant de la migraine épisodique (des patients qui présentent une céphalée migraineuse $<15$ jours par mois). Ces lignes directrices sont destinées essentiellement aux médecins qui traitent des patients migraineux. Les autres professionnels de la santé peuvent également en tirer profit. Le choix du traitement de la crise aiguë de migraine est basé sur des preuves de son efficacité et de sa tolérabilité, sur la sévérité des crises de migraine, sur les préférences du patient et sur la présence de comorbidités. Parmi les principes généraux du traitement de la crise aiguë de migraine, il est important de noter que la réponse d'un patient à un médicament particulier ne peut être prédite avec certitude et que le traitement administré tôt au cours de la crise est généralement plus efficace que le traitement administré lorsque la crise de migraine est bien installée. Une méthode de traitement convenable (méthode stratifiée ou par étapes) et la formulation du médicament (injection, comprimé, capsule, poudre ou vaporisation nasale) devraient être choisies en fonction des manifestations cliniques que présente le patient. Une fréquence excessive d'utilisation de la médication de phase aiguë (surconsommation de médicaments) est à éviter. Deux médicaments ou plus pour traiter une crise aiguë peuvent être combinés si nécessaire. Conclusions : Ces lignes directrices fournissent des conseils fondés sur des preuves pour l'utilisation de la médication pour traiter la crise aiguë de migraine et fournissent des conseils utiles sur son traitement, tant pour les professionnels de la santé que pour les patients.

Can J Neurol Sci. 2013; 40: Suppl. 3 - S4-S9

Migraine is a common neurological disorder, which can produce significant disability, and reduce health-related quality of life. ${ }^{1,2}$ Canadian studies have shown migraine prevalence rates of 23 to $26 \%$ in women, and 7.8 to $10 \%$ in men..$^{2-4}$

Over 4,000,000 Canadians suffer from migraine ${ }^{5}$, and as a result migraine is associated with a substantial social and economic impact. A study done in 1990 calculated that 7,000,000
From ${ }^{1}$ Sunnybrook Health Sciences Centre, Toronto, Ontario; ${ }^{2}$ Gladstone Headache Clinic, Toronto, Ontario; ${ }^{3}$ University of Calgary and the Hotchkiss Brain Institute, Calgary, Alberta; ${ }^{4}$ University of Western Ontario, London, Ontario; ${ }^{5}$ University of British Columbia, Vancouver, British Columbia; ${ }^{6}$ McGill University, Montreal, Quebec, ${ }^{7}$ University of Montreal, Montreal, Quebec; ${ }^{8}$ Rouge Valley Health System Centenary, Toronto, Ontario; ${ }^{9}$ Women's College Hospital, Toronto, Ontario, Canada. Received June 9, 2013. Final Revisions Submitted June 22, 2013.

Correspondence to: W.J. Becker, Division of Neurology, 12th Floor, Foothills Hospital, 1403 29th St NW, Calgary, Alberta, T2N 2T9, Canada. 
workdays were lost annually in Canada due to migraine. ${ }^{4}$ Disability related to migraine has been recognized by the World Health Organization, which ranked migraine as 19th among all causes of disability in terms of years lived with disability. ${ }^{6}$

The International Headache Society (IHS) has classified two major subtypes: migraine without aura, and migraine with aura. Migraine without aura is the most common migraine subtype, and is characterized by headache attacks lasting 4 to 72 hours. Headache attacks are usually accompanied by other symptoms including photophobia, phonophobia, nausea, and sometimes vomiting (for diagnostic criteria see Tables 1 and 2). Individuals with migraine with aura experience in addition reversible focal neurological symptoms, which usually precede the headache and last up to 60 minutes, or occasionally longer (Table 2).,

Acute (symptomatic) pharmacological migraine therapy refers to the use of medication to treat individual migraine attacks. The great majority of adults with migraine in Canada $(90 \%)$ use acute medications for their migraine attacks. ${ }^{2}$ Acute medications are, however, only one component of migraine treatment. Based on headache frequency in population studies, it would appear that up to $25 \%$ of migraine sufferers might also benefit from the use of daily preventive medications to reduce migraine frequency. ${ }^{9}$ Pharmacological prophylaxis should be considered in patients with frequent and/or refractory migraine attacks. All migraine sufferers should also consider careful management of lifestyle factors and specific migraine triggers, which can potentially increase migraine frequency (see Headache Network Canada website: http://www.headache network.ca (in English and French), Migraine Quebec website: www.migrainequebec.com (in French; to be translated into English), and American Headache Society website: http://www.americanheadachesociety.org/professionalresources/ TriggerAvoidanceInformation.asp). In addition, behavioural interventions including the mastery of relaxation techniques, stress management, pacing, cognitive behavioural therapy, and biofeedback have the potential to benefit many migraine sufferers. ${ }^{10-12}$

Table 1: International Headache Society criteria for migraine without aura $^{8}$

A. At least 5 attacks fulfilling criteria B-D

B. Headache attacks lasting 4-72 hours (untreated or unsuccessfully treated)

C. Headache has at least two of the following characteristics:

- unilateral location

- pulsating quality

- moderate or severe pain intensity

- aggravation by or causing avoidance of routine physical activity (e.g., walking or climbing stairs)

D. During headache, at least one of the following is present:

- nausea and / or vomiting

- photophobia and phonophobia

E. Not attributed to another disorder
Acute pharmacological migraine therapy includes both "migraine-specific" medications (e.g., triptans, dihydroergotamine), and "non-specific" medications (e.g., ASA, acetaminophen, NSAIDs). It also includes adjunctive drugs such as anti-emetics (e.g., domperidone, metoclopramide, prochlorperazine) in oral or rectal formulations. The introduction of sumatriptan subcutaneous injection in 1991 represented a significant advance in the management of migraine. When the first Canadian migraine guidelines were published in 1997, the only triptan available was sumatriptan. ${ }^{7}$ Since that time, six more triptans have become available to Canadians. Although triptans are generally considered to be the most effective of the acute migraine medications overall, as recently as 2005 , only $8 \%$ of Canadians listed a triptan as their main migraine medication. ${ }^{2}$ Under-utilization of effective acute therapies has the potential to negatively impact quality of life for migraine sufferers. Population-based data in 2005 indicated that at least 200,000 Canadian women with migraine were very unsatisfied with the effectiveness of their acute migraine medications. ${ }^{2,13}$

These guidelines have been developed to assist both health professionals and patients to develop more effective acute migraine treatment strategies.

\section{Table 2: International Headache Society criteria for typical} migraine with aura*8

A. At least 2 attacks fulfilling criteria B-D

B. Aura consisting of at least one of the following, but no motor weakness:

1. fully reversible visual symptoms including positive features (e.g., flickering lights, spots or lines) and/or negative features (i.e., loss of vision)

2. fully reversible sensory symptoms including positive features (i.e., pins and needles) and/or negative features (i.e., numbness)

3. fully reversible dysphasic speech disturbance

C. At least two of the following:

1. homonymous visual symptoms and/or unilateral sensory symptoms

2 . at least one aura symptom develops gradually over $\geq 5$ minutes and/or different aura symptoms occur in succession over $\geq 5$ minutes

3. each symptom lasts $\geq 5$ and $\leq 60$ minutes

D. Headache fulfilling criteria B-D for Migraine without aura begins during the aura or follows aura within 60 minutes

E. Not attributed to another disorder

* Other less common types of migraine with aura include typical aura with non-migraine headache, typical aura without headache, familial hemiplegic migraine, and others. 


\section{Guideline Objectives and Target Population}

\section{Objectives}

The primary objective of this guideline is to assist the physician in choosing an appropriate acute medication for an individual with migraine, based on current evidence in the medical literature. An additional objective is to assist the practitioner in using the chosen medication in the most effective manner.

The main clinical question that this guideline aims to help answer for the medical practitioner is, "Which acute medication should be prescribed for an individual patient in a specific clinical situation?"

The ultimate purpose or goal of this guideline is to reduce the headache-related disability suffered by individuals with migraine.

\section{Target population}

This guideline provides evidence-based recommendations for the outpatient acute management of adults (18 years-of-age or older) with episodic migraine (patients who experience migraine headache attacks on less than 15 days/month). It does not include recommendations for pediatric patients and for the emergency room management of acute migraine.

Although it is likely that physicians will extrapolate from the evidence presented here and use it for the care of patients with chronic migraine (headache on 15 days a month or more, with diagnostic criteria for migraine met on at least eight days a month), many of the clinical trials reviewed for this guideline did not include patients with headache frequencies of this magnitude.

\section{Who should use this guideline?}

This guideline is intended primarily for physicians who treat patients with migraine, including both family physicians, and specialists. Other health professionals who treat patients with migraine may also find this guideline helpful. As migraine is a chronic disorder, and it is important that patients with migraine partner with their health care professionals in order to achieve the best management success possible, individuals with migraine and their families may also find this guideline useful.

\section{Expert consensus and recommendations}

The core of this guideline is Section 2 "Targeted Review: Medications for Acute Migraine Treatment". The recommendations in this section are based on a targeted review as described in that section. Evidence from randomized controlled trials is not available, however, to guide clinicians with regard to all the clinical decisions that must be made. To recognize this, treatment suggestions made in other sections of this guideline are labeled as "expert consensus", as they are based on a general literature review and on the expert opinion of clinicians experienced in migraine treatment. These expert opinions were developed through expert consensus groups (See Appendix 1).

\section{Goals of Acute Migraine Therapy}

The goals of acute (or symptomatic) migraine therapy are to relieve pain and the associated symptoms of migraine (e.g., nausea, vomiting, photophobia, phonophobia) rapidly and consistently, with minimal or no adverse events, and to relieve migraine-related disability so that the patient can return quickly to normal function. ${ }^{7}$ Although some patients may be able to achieve the goal of becoming pain-free within two hours of taking an acute medication, those who only achieve partial headache relief (pain reduction) should try at least several acute medications (including several triptans if not contraindicated) over time for different migraine attacks, to determine if it is possible for them to reach this goal.

\section{General Principles of Acute Migraine Therapy}

\section{The response of the individual with migraine to a specific acute drug cannot be predicted with certainty}

Response to acute medications is individual and idiosyncratic. $^{7}$ If the response to the first medication is not excellent, several medications may need to be tried (in succession) over time for different attacks to determine the most suitable medication in terms of efficacy and tolerability. Access to two different medications may be necessary if a patient suffers from attacks of varying severities. The choice of a particular symptomatic medication should take into account the efficacy of past treatments, and the presence of any concomitant disorders that may preclude use of certain medications. For patients with severe attacks, a rescue medication may be needed if their usual medication fails.

\section{EXPERT CONSENSUS}

i. Several acute medication trials may be necessary before an appropriate acute medication is found for a specific patient. Some patients with attacks of varying severity may need access to more than one medication for successful migraine management.

ii. A rescue plan should be discussed with patients with severe migraine attacks whose usual acute medication does not provide adequate headache relief consistently for every attack.

\section{Early intervention: Most patients should be encouraged to take their acute medication early in the attack}

To experience maximum effectiveness, patients should use acute medications as early as possible after headache onset, and while the pain is still mild. For the triptans, several prospective studies have shown improved efficacy with early treatment (see Table 6 in Section 2). Triptans may be more effective when taken early in the attack, because they can prevent but not reverse central sensitization. Central sensitization, as manifested by cutaneous allodynia, may occur in up to $75 \%$ of patients within 20 to 60 minutes of migraine onset. ${ }^{14}$

When discussing early treatment, patients should be educated with regard to the effects of medication overuse, and the need to differentiate migraine attacks from tension-type headache. For patients with frequent migraine attacks, early treatment may need to be used very cautiously. More effective acute treatment may result in less symptomatic medication use; however, 
indiscriminate use of early treatment has the potential to lead to acute medication overuse in some patients. ${ }^{15}$ Patients may find taking acute medication during the migraine aura useful to manage their headaches, but for triptans there is evidence that they are best taken at the onset of head pain rather than during the aura. This is particularly true for subcutaneous sumatriptan (see Section 3).

\section{EXPERT CONSENSUS}

i. Patients should be advised to take acute medications as early as possible during their migraine attacks while pain is still mild, unless at risk for medication overuse headache.

\section{An appropriate treatment approach should be chosen}

Three basic treatment strategies have been documented as options for acute migraine treatment. ${ }^{16}$ In Section 3 of this guideline, we use the term "strategy" for acute treatment paradigms for specific patient groups, based on patient clinical features. To avoid confusion, we will therefore use the term "approach" rather than "strategy" for the more broad or generalized concepts of stratified and step care approaches to care, while recognizing that these were termed "strategies" in the original publications that described them:

a. "Stratified care": The medication chosen for a patient is based on attack severity and/or degree of migraine-related disability. ${ }^{17,18}$

b. "Step care within an attack": A simple analgesic or NSAID is used initially for a migraine attack. If the first medication is not successful, another medication (e.g., a triptan), is used a few hours later.

c. "Step care across attacks": The practitioner prescribes an initial medication (e.g., NSAID), and the patient tries this for several attacks. If this medication is not sufficiently effective, the practitioner would prescribe another medication (e.g., a triptan) for subsequent attacks.

In practice, many patients have tried several non-prescription medications prior to consulting a physician for their headaches; therefore, a "step care across attacks" approach is already in place when the physician prescribes a more effective medication.

"Stratified care" is likely to be the most effective acute treatment approach, and has been shown to be cost effective. ${ }^{19} \mathrm{It}$ has been promoted by several guidelines (The U.S. Headache Consortium $^{20}$, and the European Federation of Neurological Societies $^{21,22}$ ). Stratified care is the model of care recommended here for patients with the most severe migraine attacks, while a modified or "hybrid" model of care, which incorporates features of both stratified care and the "step care across attacks" model, is recommended for most patients with migraine in this guideline (see Section 3). "Step care within an attack" has been promoted by some guidelines, but it has the disadvantage that if the first medication fails, the second presumably more effective medication may fail as well, because it is taken later in the attack (at a time when it may no longer be as effective as it could have been if taken earlier). The "step care across attacks" approach has the potential disadvantage that if the initial medication provided by the physician is ineffective, patients may become discouraged and not pursue additional medical care for their migraine (become a "lapsed consulter"). If this approach is chosen, patients should be informed early of the remaining treatment options, so that they realize other therapies are available for their migraine should that prove necessary.

It needs to be recognized that many patients with migraine have more than one attack severity. If they are able to identify early in the attack whether they are going to experience a severe attack or one of lesser intensity, they may be able to choose an appropriate medication for their attack based on the stratified care model. If patients are unable to identify the ultimate severity of their migraine attack early in its course, a "step care within an attack" approach may be appropriate if the majority of their attacks are relatively mild and respond to an NSAID or other medication, and if their more severe attacks still respond to their second medication even when taken later in the attack.

\section{EXPERT CONSENSUS}

$i$. When recommending an acute migraine medication, consideration should be given to attack severity ("stratified care" approach) and past response to medications.

ii. If a "step care across attacks" approach is chosen, patients should be educated with regard to remaining available treatment options, to reduce the risk of patients becoming discouraged and no longer consulting for their headaches.

iii. Although a "step care within an attack" approach may be suitable for some patients, patients should be advised that most acute medications are more effective if taken early in the migraine attack.

\section{A suitable medication formulation should be chosen}

Patient preference needs to be considered when recommending a particular medication formulation. ${ }^{23,24}$ However, some formulations have advantages over others in specific clinical situations. For some patients, it may be advantageous to use one formulation for some attacks, and another formulation for others.

a. For migraine attacks that build up very rapidly and/or are characterized by early vomiting, and for attacks that present full-blown upon awakening, an injectable formulation (e.g., subcutaneous sumatriptan) has the potential to be most effective..$^{25-27}$

b. Patients with nausea and those who vomit only later in the attack may find nasal spray formulations to be more helpful than oral formulations, as medications delivered by nasal spray are partially absorbed through the nasal mucosa. ${ }^{23,24,28,29}$

c. Patients with lesser degrees of nausea or nausea that is exacerbated by taking water, and those who wish to treat their attacks early in situations where water may not be readily available, may find the orally disintegrating tablets more useful than regular tablets which are swallowed. Orally disintegrating tablets do not have a faster onset of action, as they are not absorbed through the buccal mucosa, but rather are swallowed with saliva, and absorbed in gastrointestinal tract. ${ }^{23,24,30}$

d. For patients without significant nausea, regular oral 
tablets, orally disintegrating tablets, nasal sprays and injections are all appropriate options. The injection formulation has the greatest efficacy, but higher cost and more discomfort. ${ }^{26,27}$ Because of partial nasal absorption, nasal spray formulations may have a slightly faster onset of action than tablets. ${ }^{28,29}$ The evidence for significant absorption through the nasal mucosa is strongest for zolmitriptan nasal spray. ${ }^{31,32}$

e. Some oral formulations are designed for faster drug delivery and onset of action, as compared to regular oral tablets. Examples are diclofenac powder for oral solution, sumatriptan DF (fast dissolving) tablet, and others (e.g., effervescent ASA, liquid-containing NSAID preparations). Patients should be made aware of these options where appropriate, so that those with migraine attacks that increase rapidly in intensity can take advantage of these special formulations if they wish to.

\section{EXPERT CONSENSUS}

$i$. When choosing an acute migraine medication for a specific patient, consideration should be given to the clinical features of the attack including rate of increase of headache intensity and the presence of nausea and / or vomiting early in the attack, and an appropriate medication formulation should be chosen. Some patients may require more than one formulation.

\section{Medication overuse needs to be avoided because of the risk of medication overuse headache}

All the commonly used acute medications have the potential to cause medication overuse headache $(\mathrm{MOH})$ in patients with migraine when used too frequently over a period of several months or more. ${ }^{33-35}$ To avoid $\mathrm{MOH}$, commonly accepted recommendations are to:

a. Limit use of acetaminophen, ASA, and NSAIDs to a maximum of 14 days a month. ${ }^{36,37}$

b. Limit use of triptans, ergotamine, opioids and combination analgesics to a maximum of 9 days a month..$^{36,37}$

For patients taking medications from both classes, the important principle is for the patient to be free of acute medications at least 20 days a month..$^{36,37}$ The International Headache Society (IHS) Diagnostic Criteria indicate that patients taking both triptans and NSAIDs should limit their use of these to a total of 9 days a month to avoid risk of $\mathrm{MOH}$ (http://ihs-classification.org/en/01_einleitung/03_anleitung/).

For patients with frequent attacks who are at risk of $\mathrm{MOH}$, behavioural approaches to migraine management and prophylactic medications should be considered in addition to acute medications.

\section{EXPERT CONSENSUS}

$i$. When initiating treatment with acute migraine medications, the patient should be educated with regard to medication overuse headache. Patients should avoid use of ASA, NSAIDs and acetaminophen on more than 14 days per month, and use of triptans, ergots, opioids, or combination analgesics on more than 9 days a month. Patients taking different acute medications on different days should limit their total use of acute medications to 9 days a month if one of their medications is a triptan, a combination analgesic, an ergotamine, or an opioid.

ii. Patients should be advised to monitor their acute medication use if their attacks are frequent, preferably with a headache diary, in order to reduce the risk of medication overuse headache.

iii. Pharmacological prophylaxis should be considered for patients with frequent migraine attacks who may be at risk of medication overuse.

\section{Two or more acute medications can be combined if necessary}

Some patients may obtain better migraine attack relief if they take two or more acute medications simultaneously for their migraine attacks. For many patients, triptans satisfactorily treat migraine related nausea as well as the headache. Others, however, may benefit from taking an anti-nauseant (e.g., metoclopramide $10 \mathrm{mg}$ ) with their triptan. Some patients with attacks that do not respond satisfactorily to a triptan alone may have better relief if they take an NSAID (e.g., naproxen sodium $550 \mathrm{mg}$ ) with their triptan. These acute treatment options are discussed in more detail in Section 3 of the guideline.

\section{EXPERT CONSENSUS}

$i$. Although a single acute medication may relieve migraine attacks satisfactorily for many patients, others may benefit from taking more than one medication simultaneously (e.g., an NSAID with an anti-nauseant; an anti-nauseant with a triptan, or a triptan with an NSAID).

\section{Choosing an Acute Migraine Medication}

There is no ideal acute migraine medication. Practitioners should find the principles of acute migraine therapy outlined above helpful in choosing an acute medication for a specific patient. Medication cost has not been directly considered in the recommendations in this guideline, although it is considered to some extent in the "combined acute medication treatment approach" in Section 3. In this approach, unless the patient has severe attacks and fits into the "stratified care" approach, less expensive NSAIDs are tried before a triptan is chosen (if necessary) as the patient's primary acute medication.

In addition, other considerations in choosing an acute medication for a specific patient include:

1. Efficacy: How strong is the evidence that the drug is effective in acute migraine therapy and how effective is it compared to other treatment options?

2. Drug side effect profile: How safe is the drug, and how well tolerated?

3. Co-existing medical and/or psychiatric disorders: Does the patient have another disorder that is a contraindication for some of the acute migraine medications (e.g., a history of peptic ulcer, or cardiovascular disease)?

4. Patient preference: Section 2 provides information on the evidence for efficacy and side effects of the various acute migraine medications. Further guidance in choosing an acute medication for a specific patient is given in Section 3. A patient survey has indicated that an overwhelming 
majority of patients consider complete relief of head pain, no recurrence, and rapid onset of action as important or very important attributes of acute migraine therapy. ${ }^{38}$ Fortunately, many clinical trials use endpoints relevant to these preferences, and these are used in this guideline where possible.

\section{REFERENCES}

1. Becker WJ, Gladstone JP, Aube M. Migraine prevalence, diagnosis, and disability. Can J Neurol Sci. 2007;34:S3-9.

2. Cooke LJ, Becker WJ. Migraine prevalence, treatment and impact: the Canadian women and migraine study. Can J Neurol Sci. 2010;37:580-7.

3. O'Brien B, Goeree R, Streiner D. Prevalence of migraine headache in Canada: a population-based survey. Int J Epidemiol. 1994;23: 1020-6.

4. Pryse-Phillips W, Findlay H, Tugwell P, Edmeads J, Murray TJ, Nelson RF. A Canadian population survey on the clinical, epidemiologic and societal impact of migraine and tension-type headache. Can J Neurol Sci. 1992;19:333-9.

5. Becker WJ, Christie SN, Mackie G, Cooper P, Canadian Headache Society Migraine Strategy Task,Force. Consensus statement: the development of a national Canadian Migraine Strategy. Can J Neurol Sci. 2010;37:449-56.

6. Leonardi M, Steiner TJ, Scher AT, Lipton RB. The global burden of migraine: measuring disability in headache disorders with WHO's Classification of Functioning, Disability and Health (ICF). J Headache Pain. 2005;6:429-40.

7. Pryse-Phillips WE, Dodick DW, Edmeads JG, et al. Guidelines for the diagnosis and management of migraine in clinical practice. Canadian Headache Society. [see comment] [erratum appears in CMAJ 1997 Nov 15;157(10):1354]. [Review] [160 refs]. CMAJ. 1997; $156: 1273-87$

8. Headache Classification Subcommittee of the International Headache, Society. The International Classification of Headache Disorders: 2nd edition. Cephalalgia. 2004;24:9-160.

9. Lipton RB, Stewart WF, Diamond S, Diamond ML, Reed M. Prevalence and burden of migraine in the United States: data from the American Migraine Study II. Headache. 2001;41: 646-57.

10. Sauro KM, Becker WJ. Multidisciplinary treatment for headache in the Canadian healthcare setting. Can J Neurol Sci. 2008;35: 46-56.

11. Rains JC, Penzien DB, McCrory DC, Gray RN. Behavioral headache treatment: history, review of the empirical literature, and methodological critique. Headache. 2005;45:S92-109.

12. Pringsheim T, Davenport WJ, MacKie G, et al. Introduction to the guideline, and general principles of migraine prophylaxis Section i. Can J Neurol Sci. 2012;39:S3-7.

13. Becker WJ, Gawel M, Mackie G, South V, Christie SN. Migraine treatment. Can J Neurol Sci. 2007;34:S10-9.

14. Burstein R, Cutrer MF, Yarnitsky D. The development of cutaneous allodynia during a migraine attack clinical evidence for the sequential recruitment of spinal and supraspinal nociceptive neurons in migraine. Brain. 2000;123:1703-9.

15. Moschiano F, D'Amico D, Allais G, et al. Early triptan intervention in migraine: an overview. [Review] [20 refs]. Neurol Sci. 2005;26:s108-10.

16. Lipton RB, Stewart WF, Stone AM, Lainez MJ, Sawyer JP, Disability in Strategies of Care Study,group. Stratified care vs step care strategies for migraine: the Disability in Strategies of Care (DISC) Study: A randomized trial. [see comment]. JAMA. 2000;284:2599-605.

17. Lipton RB, Bigal ME, Amatniek JC, Stewart WF. Tools for diagnosing migraine and measuring its severity. Headache 2004:44:387-98.
18. Lipton RB, Stewart WF, Sawyer J, Edmeads JG. Clinical utility of an instrument assessing migraine disability: the Migraine Disability Assessment (MIDAS) questionnaire. Headache. 2001; 41:854-61.

19. Williams P, Dowson AJ, Rapoport AM, Sawyer J. The cost effectiveness of stratified care in the management of migraine. Pharmacoeconomics. 2001;19:819-29.

20. Silberstein SD. Practice parameter: evidence-based guidelines for migraine headache (an evidence-based review): report of the Quality Standards Subcommittee of the American Academy of Neurology. Neurology. 2000;55:754-62.

21. Evers S, Afra J, Frese A, et al. EFNS guideline on the drug treatment of migraine - report of an EFNS task force. Eur $\mathbf{J}$ Neurol 2006;13:560-72.

22. Evers S, Afra J, Frese A, et al. EFNS guideline on the drug treatment of migraine--revised report of an EFNS task force. Eur J Neurol. 2009;16:968-81

23. Gladstone JP, Gawel M. Newer formulations of the triptans: advances in migraine management. [Review] [116 refs]. Drugs. 2003;63:2285-305.

24. Rapoport AM, Tepper SJ, Bigal ME, Sheftell FD. The triptan formulations : how to match patients and products. [Review] [75 refs]. CNS Drugs. 2003;17:431-47.

25. Anonymous. Treatment of migraine attacks with sumatriptan. The Subcutaneous Sumatriptan International Study Group. N Engl J Med. 1991;325:316-21.

26. Tfelt-Hansen P, De Vries P, Saxena PR. Triptans in migraine: a comparative review of pharmacology, pharmacokinetics and efficacy. Drugs. 2000;60:1259-87.

27. Gawel MJ, Worthington I, Maggisano A. A systematic review of the use of triptans in acute migraine.[see comment]. [Review] [74 refs]. Can J Neurol Sci. 2001;28:30-41.

28. Gawel M, Worthington I. Intranasal zolmitriptan. Expert Opin Pharmacother. 2005;6:1019-24.

29. Rapoport A, Winner P. Nasal delivery of antimigraine drugs: clinical rationale and evidence base. Headache. 2006;46:S192201.

30. Johnston MM, Rapoport AM. Triptans for the management of migraine. Drugs. 2010;70:1505-18.

31. Yates R, Sörensen J, Bergström M, et al. Distribution of intranasal C-zolmitriptan assessed by positron emission tomography. Cephalalgia. 2005 Dec;25(12):1103-9.

32. Kagedal M, Zingmark P-, Hedlund C, Yates R. True nasopharyngeal absorption of zolmitriptan after administration via nasal spray in healthy male volunteers. American Journal of Drug Delivery. 2005;3:133-40

33. Dodick D, Freitag F. Evidence-based understanding of medicationoveruse headache: clinical implications. Headache. 2006;46: S202-11.

34. Tepper SJ, Tepper DE. Breaking the cycle of medication overuse headache. Cleve Clin J Med. 2010;77:236-42

35. Evers S, Marziniak M. Clinical features, pathophysiology, and treatment of medication-overuse headache. Lancet Neurology. 2010;9:391-401.

36. Silberstein SD, Olesen J, Bousser MG, et alInternational Headache Society. The International Classification of Headache Disorders, 2nd Edition (ICHD-II)--revision of criteria for 8.2 Medicationoveruse headache. Cephalalgia. 2005;25:460-5.

37. Headache Classification Committee. Olesen J, Bousser MG, Diener $\mathrm{HC}$, et al. New appendix criteria open for a broader concept of chronic migraine. Cephalalgia. 2006;26:742-6.

38. Lipton RB, Hamelsky SW, Dayno JM. What do patients with migraine want from acute migraine treatment? Headache. 2002; 42:3-9. 